\title{
Vigilância entomológica dos vetores da doença de Chagas nos municípios da VIII Gerência Regional de Saúde do estado de Pernambuco, Brasil, de 2012 a 2017
}

\section{Entomological surveillance of Chagas disease vectors in the municipalities of the VIII Regional Health Management of Pernambuco State, Brazil, from 2012 to 2017}

\author{
Luís Ricardo Soares da Silva'(D), Maria Beatriz Araújo Silva²,3iD, Gênova Maria de Azevedo Oliveira ${ }^{4}$ (D), Carolina de \\ Araújo Medeiros ${ }^{5}$ (D), Jaqueline Bianque de Oliveira ${ }^{1,6}$ (iD \\ Universidade Federal Rural de Pernambuco, Departamento de Biologia, Laboratório de Parasitologia, Recife, Pernambuco, Brasil \\ 2 Secretaria Estadual de Saúde de Pernambuco, Laboratório Central de Saúde Pública "Dr. Milton Bezerra Sobral", Recife, Pernambuco, Brasil \\ ${ }^{3}$ Universidade de Pernambuco, Faculdade de Enfermagem, Recife, Pernambuco, Brasil \\ ${ }^{4}$ Secretaria Estadual de Saúde de Pernambuco, Recife, Pernambuco, Brasil \\ ${ }^{5}$ Universidade de Pernambuco, Pronto Socorro Cardiológico de Pernambuco, Ambulatório de Doença de Chagas e Insuficiência Cardíaca, Recife, \\ Pernambuco, Brasil \\ - Universidade Federal Rural de Pernambuco, Programa de Pós-Graduação em Biociência Animal, Recife, Pernambuco, Brasil
}

\section{RESUMO}

OBJETIVOS: Registrar a distribuição das espécies de triatomíneos encontradas no ambiente domiciliar, de 2012 a 2017 , na VIII Gerência Regional de Saúde (GERES) do estado de Pernambuco, Brasil, e identificar a taxa de infecção natural por flagelados morfologicamente semelhantes a Trypanosoma cruzi nesses insetos. MATERIAIS E MÉTODOS: Foram utilizadas informações do banco de dados do Programa de Controle da Doença de Chagas de Pernambuco, referentes aos triatomíneos coletados nos domicílios dos municípios, identificados e examinados no laboratório da VIII GERES. Apenas os insetos com dados de origem e de infecção natural foram considerados. A taxa de infecção natural foi calculada utilizando indicadores entomológicos. RESULTADOS: Foram coletados 9.738 triatomíneos de seis espécies: Triatoma brasiliensis (8.251), Triatoma pseudomaculata (1.323), Panstrongylus lutzi (100), Triatoma sordida (56), Panstrongylus megistus (sete) e Rhodnius neglectus (um). A taxa de infecção para flagelados morfologicamente semelhantes a T. cruzi foi 2\%. Petrolina, maior município da VIII GERES, apresentou o maior número de espécimes (3.420) e Dormentes apresentou a maior taxa de triatomíneos infectados (3,3\%). Apenas T. brasiliensis foi positiva para a infecção em todos os municípios, enquanto P. lutzi apresentou a maior taxa de infecção (38,0\%). Em 2014, foi encontrado o maior número de triatomíneos (3.186), enquanto, em 2012, a maior quantidade de insetos infectados (67), com o agente etiológico da doença de Chagas (5,0\%). CONCLUSÃO: O presente estudo contribui com a vigilância em saúde, uma vez que foram encontradas espécies positivas em todos os municípios da VIII GERES, sinalizando o risco de transmissão vetorial.

Palavras-chave: Triatomíneos; Trypanosoma cruzi; Infecção Natural.

\begin{abstract}
OBJECTIVES: To register the distribution of triatomine species found in the home environment, from 2012 to 2017 , in the VIII Regional Health Management (GERES) of Pernambuco State, Brazil, and identify the natural infection rate by flagellates morphologically similar to Trypanosoma cruzi on these insects. MATERIALS AND METHODS: Information from the Pernambuco Chagas Disease Control Program database was used, referring to triatomines collected in the homes of the municipalities, identified, and examined in the laboratory of the VIII GERES. Only insects with data of origin and natural infection were considered. The natural infection rate was calculated using entomological indicators. RESULTS: A total of 9,738 triatomines of six species were collected: Triatoma brasiliensis (8,251), Triatoma pseudomaculata (1,323), Panstrongylus lutzi (100), Triatoma sordida (56), Panstrongylus megistus (seven), and Rhodnius neglectus (one). The infection rate for flagellates morphologically similar to T. cruzi it was $2 \%$. Petrolina, the largest municipality in the VIII GERES, had the biggest number of specimens $(3,420)$ and Dormentes had the highest rate of infected triatomines $(3.3 \%)$. Only T. brasiliensis was positive for the infection in all municipalities, while $P$. lutzi had the highest infection rate (38.0\%). In 2014, the biggest number of triatomines $(3,186)$ was found, while 2012 had the most infected insects $(67)$ with the etiological agent of Chagas disease (5.0\%). CONCLUSION: This study contributes to health surveillance, since positive species were found in all municipalities of the VIII GERES, signaling the risk of vector transmission.
\end{abstract}

Keywords: Triatomines; Trypanosoma cruzi; Natural Infection.

\section{Correspondência / Correspondence:}

Luís Ricardo Soares da Silva

Universidade Federal Rural de Pernambuco, Departamento de Biologia, Laboratório de Parasitologia

Rua Dom Manoel de Medeiros, s/n. Bairro: Dois Irmãos. CEP: 52171-900-Recife, Pernambuco, Brasil

E-mail: 22luis.ricardo@gmail.com 


\section{INTRODUÇÃO}

A doença de Chagas, também conhecida como tripanossomíase americana, tem como agente etiológico o protozoário flagelado Trypanosoma cruzi ${ }^{1}$, que é transmitido primariamente por insetos vetores, os triatomíneos, que são insetos hematófagos, pertencentes à ordem Hemiptera, subordem Heteroptera, família Reduviidae, subfamília Triatominae 2,3,4 $^{\text {. }}$

De acordo com a Organização Mundial da Saúde (OMS) ${ }^{5}$, cerca de 6 a 7 milhões de pessoas estão infectadas com T. cruzi no mundo, sendo a doença de Chagas encontrada em áreas endêmicas de 21 países da América Latina; entretanto, não ocorre nas ilhas do Caribe. No Brasil, foram registrados 4.287 óbitos decorrentes dessa doença no ano de 2019, com 1.937 ocorridos na Região Sudesté ${ }^{6}$ Segundo o Ministério da Saúde7, de 2012 a 2016, foram confirmados 1.190 casos agudos da doença de Chagas, com a Região Norte apresentando a maior quantidade de casos, $97,1 \%$.

No mundo, iá foram descritas 155 espécies de triatomíneos, agrupadas em 18 gêneros e cinco tribos, sendo 68 espécies conhecidas no Brasil $7,8,9$.

A Região Nordeste brasileira é considerada de importância epidemiológica ${ }^{10}$, por apresentar grandes focos de transmissão ${ }^{11}$, e está dividida em regiões fisiográficas distintas que envolvem uma diversidade de 29 espécies de triatomíneos ${ }^{8,10}$, sendo Triatoma brasiliensis e Triatoma pseudomaculata consideradas espécies nativas da região ${ }^{12}$. Em diversos municípios dessa região, a população apresenta uma alta prevalência da infecção por T. cruzi ${ }^{11}$.

No estado de Pernambuco, já foram encontradas 14 espécies de triatomíneos: Panstrongylus lutzi Neiva \& Pinto, 1923; Panstrongylus megistus Burmeister, 1835; Rhodnius nasutus Stål, 1859; Rhodnius neglectus Lent, 1954; T. brasiliensis Neiva, 1911; Triatoma melanocephala Neiva \& Pinto, 1923; Triatoma petrochiae Pinto \& Barreto, 1925; T. pseudomaculata Correa \& Espínola, 1964; Triatoma rubrofasciata (De Geer, 1773); Triatoma sordida (Stål, 1859); Triatoma tibiamaculata (Pinto, 1926); Rhodnius tertius (Lent \& Jurberg, 1966); Panstrongylus geniculatus (Latreille, 1811); e Triatoma infestans (Klug, 1834). Esta última espécie foi eliminada do Estado após intensas atividades de controle, que culminaram na certificação da interrupção de transmissão vetorial pela mesma no Brasil $12,13,14,15$. As espécies T. brasiliensis e T. pseudomaculata, atualmente, se destacam como principais vetores da doença de Chagas no Estado, além de $P$. lutzi, que também é infectado naturalmente por T. cruzi ${ }^{12}$.

Recentemente, em Pernambuco, na cidade de Ibimirim, foi registrado um dos maiores surtos de doença de Chagas aguda do Brasil, associado, preliminarmente, à ingestão de alimentos contaminados, que resultou em 30 indivíduos tratados ${ }^{16}$.

Os estudos de distribuição geográfica de triatomíneos são fundamentais para a compreensão de aspectos epidemiológicos relacionados à transmissão de $T$. cruzi e devem ser considerados para orientar as ações de vigilância e controle da doença de $\mathrm{Chagas}^{13}$.

Portanto, o presente trabalho teve como objetivos registrar a distribuição das espécies de triatomíneos encontradas no ambiente domiciliar, no período entre 2012 e 2017, na VIII Gerência Regional de Saúde (GERES) de Pernambuco, que está constituída por municípios prioritários para o controle da doença de Chagas em Pernambuco, e identificar a taxa de infecção natural por flagelados morfologicamente semelhantes a T. cruzi dos respectivos insetos.

\section{MATERIAIS E MÉTODOS}

Trata-se de um estudo descritivo, quantitativo e retrospectivo, com dados entomológicos de triatomíneos coletados no período de 2012 a 2017, na VIII GERES de Pernambuco.

Pernambuco apresenta uma área territorial de $98.076,021 \mathrm{~km}^{2}$, com população estimada de 9.473.266 pessoas, densidade demográfica de 89,62 habitantes $/ \mathrm{km}^{2}$, com Índice de Desenvolvimento Humano de 0,673 ${ }^{17}$. Em todos os 184 municípios de Pernambuco e na ilha de Fernando de Noronha, foram criadas 12 GERES, unidades administrativas da Secretaria Estadual de Saúde, sendo cada uma responsável por uma parte das cidades, atuando de forma mais localizada na atenção básica, na reestruturação da rede hospitalar, nas ações municipais e no combate à mortalidade infantil e às diversas endemias.

A VIII GERES é composta por sete municípios (Figura 1), todos pertencentes ao Sertão do São Francisco: Afrânio (8³0'54"S, 4100'18"W), com população de 17.586 habitantes ${ }^{18}$; Cabrobó (8³0'51"S, 39¹8'36"W), com população de 30.873 habitantes $^{19}$; Dormentes ( $\left.8^{\circ} 26^{\prime} 50^{\prime \prime} \mathrm{S}, 40^{\circ} 46^{\prime} 16^{\prime \prime} \mathrm{W}\right)$, com população de 16.917 habitantes ${ }^{20}$; Lagoa Grande (859'49"S, 40¹6'19"W), com população de 22.760 habitantes $^{21}$; Orocó (8³7'06"S, 39³6'02"W), com população de 13.180 habitantes $^{22}$; Santa Maria da Boa Vista ( $\left.8^{\circ} 48^{\prime} 28^{\prime \prime} S, 39^{\circ} 49^{\prime} 32^{\prime \prime} \mathrm{W}\right)$, com população de 39.435 habitantes $^{23}$; e Petrolina (9²3'55"S, 4030'3"W), população de 293.962 habitantes $^{24}$, sede da VIII GERES12,25,26. Esses municípios apresentam a caatinga como vegetação primitiva ${ }^{27}$, com uma área total de $14.654,862 \mathrm{~km}^{2}$ e clima semiárido 28,29

Nos municípios da VIII GERES, o controle de triatomíneos é realizado por controle químico (uso de inseticidas), juntamente com a vigilância ativa e passiva, tanto nas áreas urbanas quanto nas áreas rurais. $\mathrm{Na}$ vigilância ativa, os agentes de combate às endemias (ACE) municipais deslocam-se até as residências e coletam os insetos, sem notificação prévia do morador, enquanto, na vigilância passiva, os próprios moradores notificam os ACE para a realização da coleta. As habitações são estruturadas com paredes de alvenaria com reboco, alvenaria sem reboco, barro com reboco, barro sem reboco ou madeira. Os tetos são constituídos por telha, palha, madeira ou material metálico. 


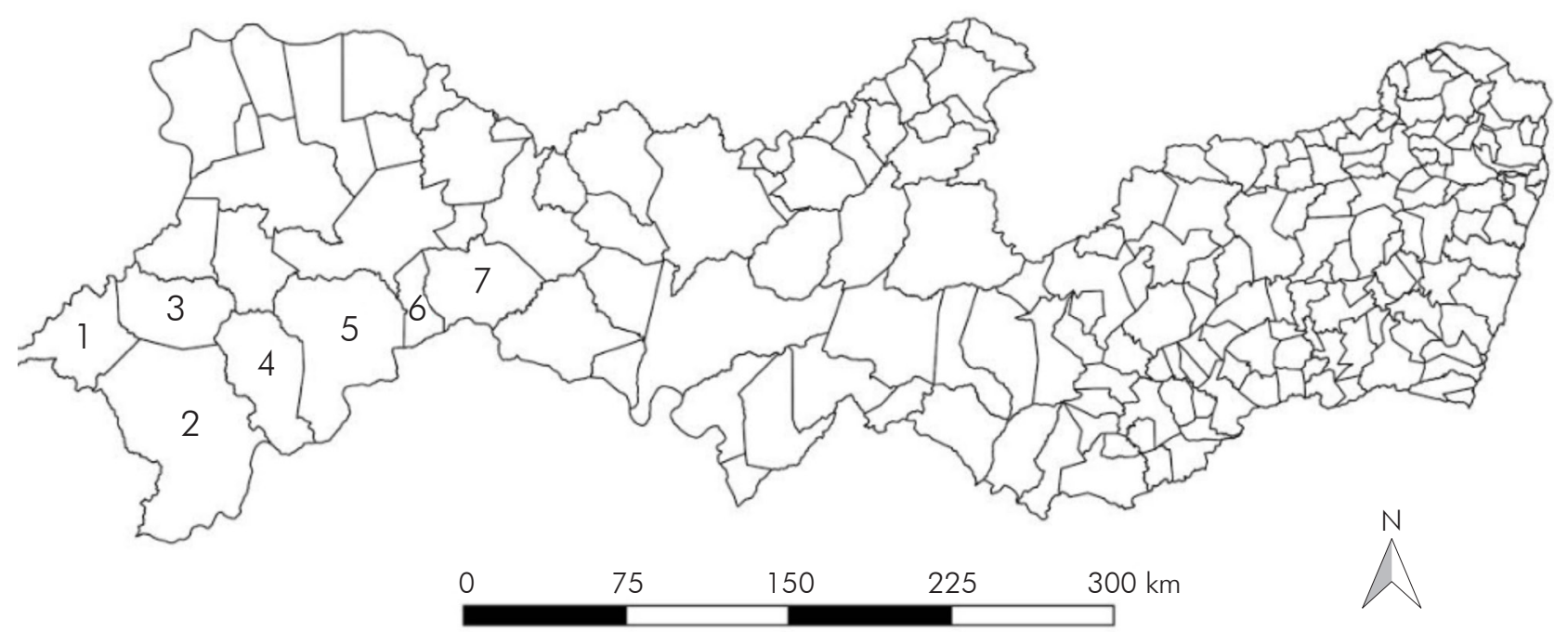

1: Afrânio; 2: Petrolina; 3: Dormentes; 4: Lagoa Grande; 5: Santa Maria da Boa Vista; 6: Orocó; 7 Cabrobó.

Figura 1 - Localização dos municípios da VIII GERES, estado de Pernambuco, Brasil

As informações utilizadas no presente estudo são provenientes do banco de dados do Programa de Controle da Doença de Chagas (SISPCDCh) do estado de Pernambuco e referentes aos triatomíneos coletados nos domicílios dos municípios, identificados e examinados no laboratório da VIII GERES, em Petrolina, e registrados no Sistema DOS-SISPCDCh.

Os insetos foram coletados pelos ACE municipais em ambiente domiciliar de áreas urbanas e rurais, utilizando pinças entomológicas e lanternas para inspeção de fendas e locais desprovidos de iluminação.

Os triatomíneos foram encaminhados para O Laboratório de Entomologia regional da VIII GERES, para identificação taxonômica e exame parasitológico de infecção por flagelados morfologicamente semelhantes a T. cruzi. Os triatomíneos foram identificados até o nível de espécie de acordo com Lent e Wygodzinsky ${ }^{30}$ e Galvão e Dale ${ }^{31}$. $O$ exame parasitológico foi realizado mediante compressão abdominal dos triatomíneos e a identificação dos parasitos morfologicamente semelhantes a T. cruzi foi efetivada em lâminas coradas com Giemsa e examinadas em microscópio óptico (400X). Foi considerado como critério de exclusão, os insetos que apresentaram incompletude dos dados de origem e de infecção natural.

A taxa de infecção natural foi calculada utilizando indicadores entomológicos preconizados pela Organização Pan-Americana da Saúde ${ }^{32}$ :

$$
\mathbb{I N}=\frac{\text { Número de triatomíneos infectados por T. cruzi x } 100}{\text { Número de triatomíneos examinados }}
$$

Foram utilizados os softwares Microsoft Excel 2010 e QGIS v3.10 para a confecção dos mapas. $\bigcirc$ estudo foi aprovado pelo Comitê de Ética em Pesquisa da Universidade de Pernambuco - CEP-PROPEGI, parecer número 3.376.395, em 6 de junho de 2019.

\section{RESULTADOS}

No período de 2012 a 2017, foram coletados 9.738 espécimes de triatomíneos pertencentes a seis espécies: T. brasiliensis (8.251), T. pseudomaculata (1.323), P. lutzi (100), T. sordida (56), P. megistus (sete) e R. neglectus (um). P. lutzi, T. brasiliensis e T. pseudomaculata foram encontradas em todos os municípios do Sertão de São Francisco. A taxa de infecção para flagelados morfologicamente semelhantes a T. cruzi nos triatomíneos examinados foi de $2 \%$. Das seis espécies coletadas, quatro foram encontradas positivas: P. lutzi, T. brasiliensis, T. pseudomaculata e T. sordida (Tabela 1).

No município de Afrânio, foram coletados espécimes de cinco espécies de triatomíneos e foi o único com registro da espécie $R$. neglectus. Chama a atenção a alta taxa de infecção de P. lutzi $(59,3 \%)$ por flagelados morfologicamente semelhantes a $T$. cruzi nesse município. $\bigcirc$ município de Dormentes apresentou quatro espécies de triatomíneos e O P. lutzi foi o que apresentou a maior taxa de infecção $(44,4 \%)$. No município de Lagoa Grande, foram encontradas as mesmas espécies coletadas em Dormentes; no entanto, as taxas totais de infecção foram diferentes: 1,0\% e 3,3\%, respectivamente. Em Petrolina, foram coletadas cinco espécies de triatomíneos, destacando-se, mais uma vez, a alta taxa de infecção de P. lutzi $(46,7 \%)$. Com relação ao município de Santa Maria da Boa Vista, cinco espécies foram identificadas, as mesmas encontradas no município de Petrolina, sendo essa a maior diversidade de espécies. P. lutzi também foi a espécie de maior taxa de infecção $(20,6 \%)$ em Santa Maria da Boa Vista. Os municípios de Cabrobó e Orocó apresentaram três espécies cada (Tabela 1).

T. brasiliensis foi a única espécie que apresentou a infecção por flagelados morfologicamente semelhantes a T. cruzi em todos os municípios estudados, enquanto T. pseudomaculata só não foi infectada em Cabrobó. A maior taxa de infecção, no geral, foi registrada em $P$. lutzi (38,0\%) (Tabela 1). 
município de Petrolina apresentou a maior quantidade de triatomíneos coletados (3.420), bem como a maior quantidade de insetos naturalmente infectados (83). No entanto, Dormentes apresentou a maior taxa de infecção (3,3\%). Orocó apresentou a menor quantidade de triatomíneos coletados (182), enquanto Cabrobó apresentou a menor taxa de infecção por flagelados morfologicamente semelhantes a T. cruzi $(0,2 \%)$. $\bigcirc$ quantitativo de insetos naturalmente infectados por flagelados morfologicamente semelhantes a T. cruzi, por município estudado, é apresentado na tabela 1 e na figura 2.

Tabela 1 - Distribuição de espécies de triatomíneos, número de positivas e taxa de infecção de protozoários morfologicamente semelhantes a T. cruzi, no ambiente domiciliar de munícipios da VIII GERES, estado de Pernambuco, Brasil, entre 2012 e 2017

\begin{tabular}{|c|c|c|c|c|c|c|c|c|c|c|c|c|c|c|c|c|c|c|c|c|c|}
\hline \multirow{4}{*}{ Municípios } & \multicolumn{21}{|c|}{ Espécies de triatomíneos } \\
\hline & \multicolumn{3}{|c|}{$\begin{array}{c}\text { Panstrongylus } \\
\text { lutzi }\end{array}$} & \multicolumn{3}{|c|}{$\begin{array}{l}\text { Panstrongylus } \\
\text { megistus }\end{array}$} & \multicolumn{3}{|c|}{$\begin{array}{l}\text { Rhodnius } \\
\text { neglectus }\end{array}$} & \multicolumn{3}{|c|}{$\begin{array}{l}\text { Triatoma } \\
\text { brasiliensis }\end{array}$} & \multicolumn{3}{|c|}{$\begin{array}{c}\text { Triatoma } \\
\text { pseudomaculata }\end{array}$} & \multicolumn{3}{|c|}{$\begin{array}{l}\text { Triatoma } \\
\text { sordida }\end{array}$} & \multicolumn{3}{|c|}{ Total por município } \\
\hline & C & $P$ & IN & C & $P$ & IN & C & $P$ & IN & C & $P$ & IN & C & $P$ & $\mathbb{I N}$ & C & $P$ & IN & C & $P$ & IN \\
\hline & $N$ & $\mathrm{~N}$ & $\%$ & N & N & $\%$ & $\mathrm{~N}$ & N & $\%$ & N & $\mathrm{N}$ & $\%$ & $\mathrm{~N}$ & $N$ & $\%$ & N & $\mathrm{N}$ & $\%$ & $\mathrm{~N}$ & $\mathrm{~N}$ & $\%$ \\
\hline Afrânio & 27 & 16 & 59,3 & 1 & - & - & 1 & - & - & 1.768 & 19 & 1,1 & 284 & 1 & 0,4 & - & - & - & 2.081 & 36 & 1,7 \\
\hline Cabrobó & 3 & - & - & - & - & - & - & - & - & 462 & 1 & 0,2 & 98 & - & - & - & - & - & 563 & 1 & 0,2 \\
\hline Dormentes & 18 & 8 & 44,4 & 1 & - & - & - & - & - & 769 & 22 & 2,9 & 149 & 1 & 0,7 & - & - & - & 937 & 31 & 3,3 \\
\hline Lagoa Grande & 1 & - & - & 2 & - & - & - & - & - & 710 & 7 & 1,0 & 99 & 1 & 1,0 & - & - & - & 812 & 8 & 1,0 \\
\hline Orocó & 2 & - & - & - & - & - & - & - & - & 168 & 2 & 1,2 & 12 & 1 & 8,3 & - & - & - & 182 & 3 & 1,6 \\
\hline Petrolina & 15 & 7 & 46,7 & 1 & - & - & - & - & - & 2.934 & 71 & 2,4 & 418 & 4 & 1,0 & 52 & 1 & 1,9 & 3.420 & 83 & 2,4 \\
\hline $\begin{array}{l}\text { Santa Maria da } \\
\text { Boa Vista }\end{array}$ & 34 & 7 & 20,6 & 2 & - & - & - & - & - & 1.440 & 22 & 1,5 & 263 & 4 & 1,5 & 4 & - & - & 1.743 & 33 & 1,9 \\
\hline Total & 100 & 38 & 38,0 & 7 & - & - & 1 & - & - & 8.251 & 144 & 1,7 & 1.323 & 12 & 0,9 & 56 & 1 & 1,8 & 9.738 & 195 & 2,0 \\
\hline
\end{tabular}

C: Coletados; P: Positivos; IN: Taxa de infecção natural; Sinal convencional utilizado: - Dado numérico igual a zero, não resultante de arredondamento.

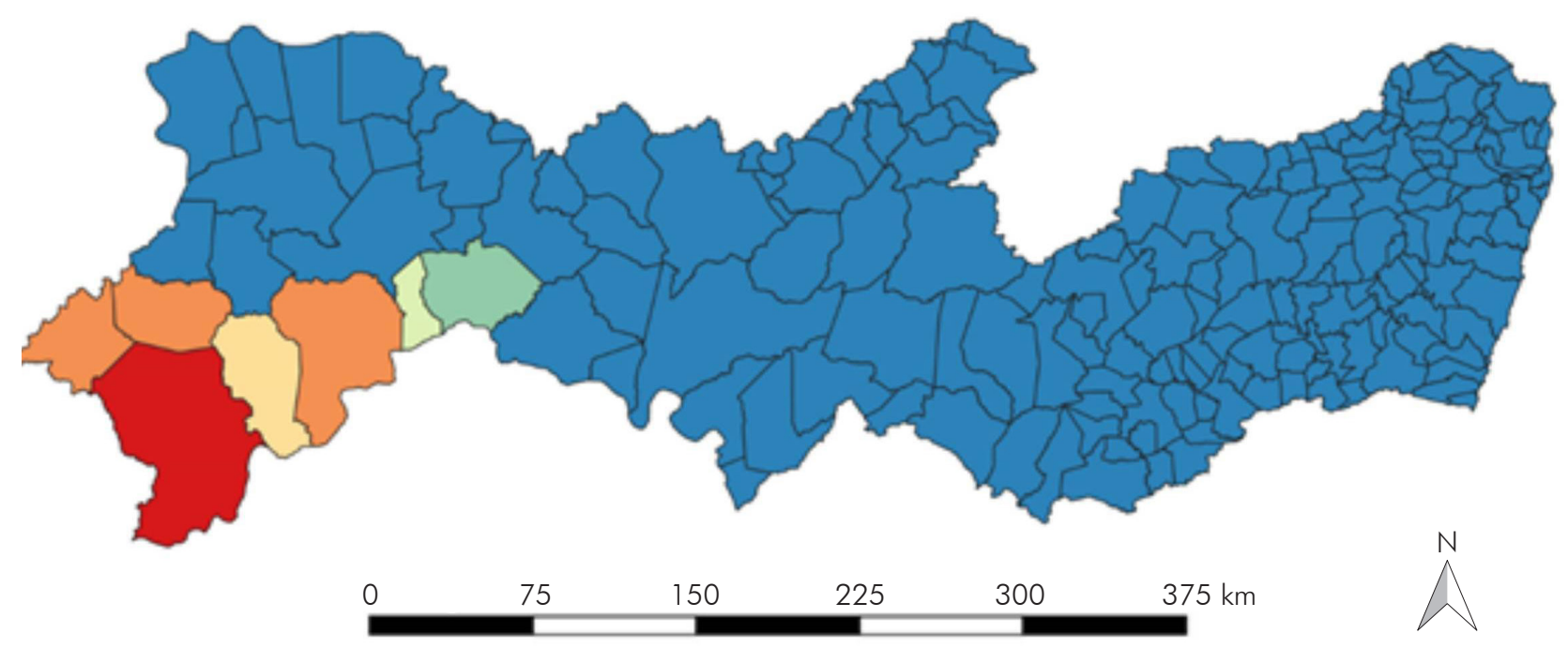

Municípios VIII GERES_TRIATOMÍNEOS
$0-1$
$1-8$
$8-21$
$21-51$
$51-83$

Em azul, os municípios pernambucanos que não fazem parte da VIII GERES.

Figura 2 - Mapa da distribuição de triatomíneos infectados por flagelados morfologicamente semelhantes a T. cruzi no ambiente domiciliar em municípios da VIII GERES, estado de Pernambuco, Brasil, entre 2012 e 2017 


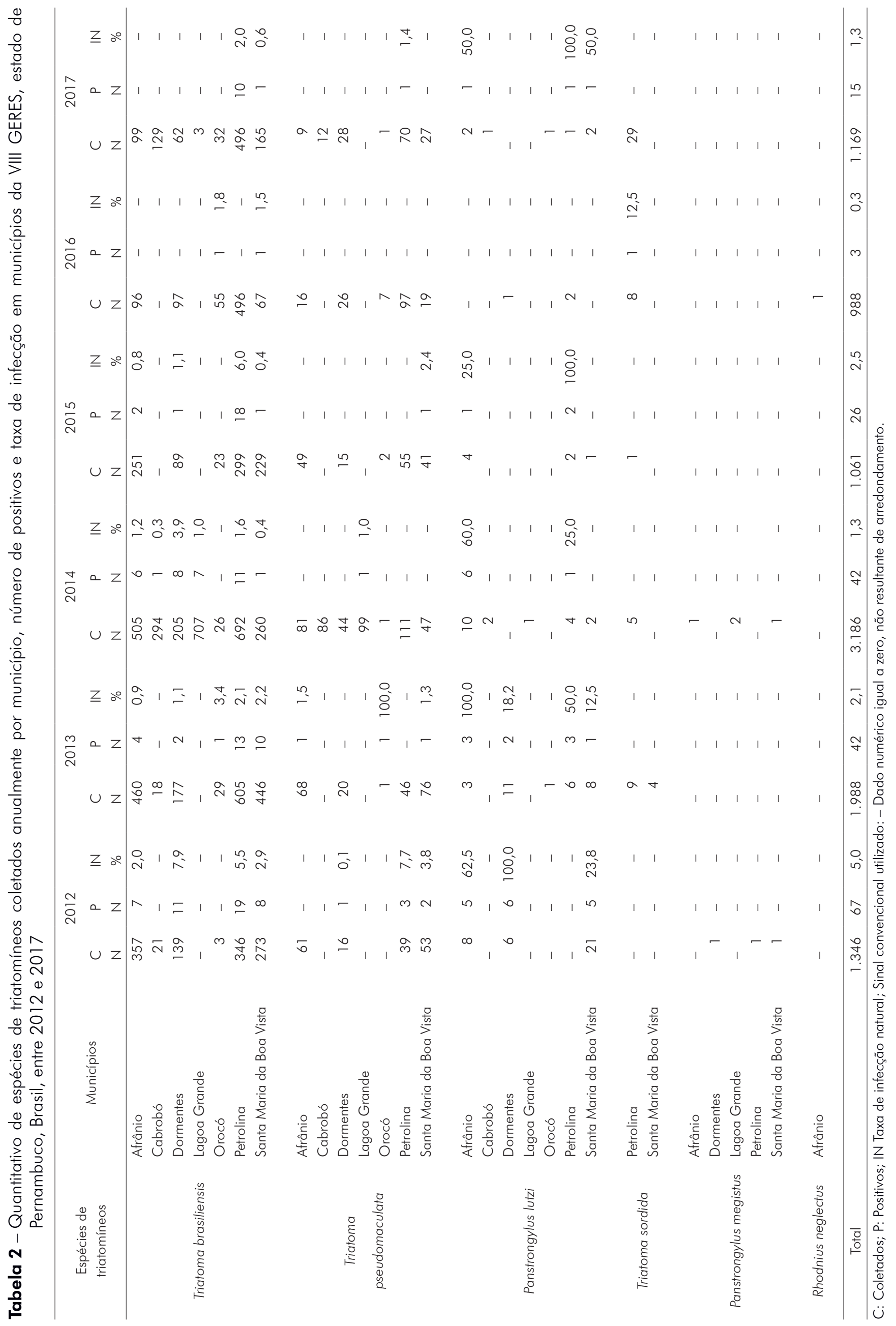


ano de 2014 foi o que apresentou a maior quantidade de triatomíneos coletados (3.186). Porém, o ano com o maior quantitativo de triatomíneos positivos (67) e a maior taxa de infecção para flagelados morfologicamente semelhantes a T. cruzi $(5,0 \%)$ foi 2012 (Tabela 2).

Em todos os anos, T. brasiliensis foi a espécie mais coletada: 2012 (357), em Afrânio; 2014 (707), em Lagoa Grande; e 2013 (605), 2015 (299), 2016 (496) e 2017 (496), em Petrolina. Durante o período de estudo, T. brasiliensis apresentou a maior positividade em Petrolina, nos anos 2012 (19), 2013 (13), 2014 (11), 2015 (18) e 2017 (10) (Tabela 2).

P. lutzi apresentou as maiores taxas de infecção por cinco anos: em 2012, 100,0\% em Dormentes; em 2013 e 2014, em Afrânio, com 100,0\% e 60,0\%, respectivamente; e em 2015 e 2017, 100,0\% em Petrolina. No ano de 2013, T. pseudomaculata apresentou a maior taxa de infecção para flagelados morfologicamente semelhantes a T. cruzi em Orocó (100,0\%). Em 2016, a maior taxa de infecção (12,5\%) foi detectada em T. sordida em Petrolina, onde a espécie só não foi encontrada em 2012 (Tabela 2).

P. megistus foi registrada, em 2012, nos municípios de Dormentes, Petrolina e Santa Maria da Boa Vista e, em 2014, nos municípios de Afrânio, Lagoa Grande e Santa Maria da Boa Vista. R. neglectus foi registrada somente no ano de 2016, em Afrânio (Tabela 2).

\section{DISCUSSÃO}

\section{OCORRÊNCIA DAS ESPÉCIES DE TRIATOMÍNEOS}

Todas as espécies identificadas no presente estudo já foram registradas em Pernambuco e são comumente encontradas em regiões semiáridas $8,13,14,15,33,34,35$. A maior diversidade de triatomíneos pode favorecer a transmissão do T. cruzi por aumentar a possibilidade da presença de espécies de importância epidemiológica ${ }^{36}$. Todas as espécies encontradas neste estudo também foram detectadas em dois municípios cearenses, Caririaçu e Jardim, os quais apresentam as mesmas características climáticas e fitogeográficas do bioma Caatinga $^{35}$.

Silva et al. ${ }^{33}$ identificaram seis espécies de triatomíneos (T. brasiliensis, T. pseudomaculata, P. lutzi, Rhodnius nasutus, T. sordida e T. infestans) na VIII GERES, em 2006 e 2007, sendo que somente quatro delas foram detectadas neste estudo (T. brasiliensis, T. pseudomaculata, P. lutzi e T. sordida), mostrando uma importante modificação na dinâmica de transmissão da doença de Chagas, pós-controle de T. infestans, importante vetor do T. cruzi.

Espécimes de T. brasiliensis e T. pseudomaculata foram encontrados por Silva et al. ${ }^{34}$, em 2012, apenas em quatro municípios: Afrânio, Petrolina, Dormentes e Santa Maria da Boa Vista; enquanto, neste estudo, foram detectados em todos os municípios da VIII GERES, demonstrando a disseminação dessas espécies. Em relação à $P$. lutzi, neste estudo, foi coletada em todos os sete municípios do Sertão de São Francisco, mas Silva et al. ${ }^{34}$ só a encontraram em Afrânio, Dormentes e Santa Maria da Boa Vista. Do mesmo modo, no presente estudo, houve registro de P. megistus em Afrânio, Dormentes, Lagoa Grande, Petrolina e Santa Maria da Boa Vista, enquanto Silva et al. ${ }^{34}$ registraram a ocorrência dessa espécie apenas em Petrolina.

Tomando como base os estudos de Silva et al. ${ }^{33,34}$, percebe-se diferenças em relação à distribuição das espécies de triatomíneos nos municípios da VIII GERES. Durante o biênio 2006-2007, seis espécies foram registradas (T. brasiliensis, T. pseudomaculata, P. lutzi, R. nasutus, T. sordida e T. infestans) ${ }^{33}$; em 2012, três delas não foram encontradas (T. sordida, $R$. nasutus e T. infestans) ${ }^{34}$. Durante o período analisado da presente pesquisa, seis espécies foram registradas, sendo três mais frequentes (T. brasiliensis, T. pseudomaculata e P. lutzi), seguidas de T. sordida.

Ressalta-se que o Brasil recebeu, em 2006, o certificado de eliminação da transmissão do T. cruzi por T. infestans, após implementação de um programa de controle de vetores ${ }^{37}$, o qual teve significativo impacto nos indicadores entomológicos dessa espécie, podendo ser um dos fatores a afetar a diversidade de espécies.

Algumas espécies, como P. lutzi, T. brasiliensis e T. pseudomaculata, foram encontradas em todos os municípios da VIII GERES, sendo o relato de maior diversidade registrado para os municípios de Afrânio, Petrolina e Santa Maria da Boa Vista. T. brasiliensis foi a espécie mais frequente, seguida por T. pseudomaculata e P. lutzi, o que também foi comprovado por Silva et al. ${ }^{33}$. Essas espécies também foram coletadas na região do Sertão de São Francisco, incluindo P. megistus, e no Agreste do estado, em 2013, com T. brasiliensis sendo a espécie mais abundante ${ }^{8,27}$.

Silva et al. ${ }^{34}$ não registraram a ocorrência de $P$. megistus e $R$. neglectus no município de Afrânio. Entretanto, T. brasiliensis foi a espécie mais frequente, o que também foi observado no presente estudo, permanecendo predominante.

No ano de 2012, Silva et al. ${ }^{34}$ não relataram a ocorrência de triatomíneos nos municípios de Cabrobó, Lagoa Grande e Orocó, resultados divergentes do presente estudo, sugerindo a hipótese de estar ocorrendo disseminação das espécies de triatomíneos na VIII GERES.

Em Petrolina, foram coletadas as espécies T. brasiliensis, T. pseudomaculata, P. megistus, T. sordida e P. lutzi, sendo que as duas últimas não foram registradas por Silva et al. ${ }^{34}$ nesse município. Por fim, em Santa Maria da Boa Vista, foram encontradas as espécies T. brasiliensis, T. pseudomaculata, P. lutzi, T. sordida, e P. megistus, com as duas últimas sem ocorrência no município no estudo de Silva et al. ${ }^{34}$.

Tendo como parâmetro os estudos realizados na mesma região por Silva et al.33,34, a flutuação da densidade pode ser observada nos diferentes municípios estudados. Uma das respostas para as mudanças na ocorrência das espécies, ao longo dos anos da presente pesquisa, se deve ao comportamento instável dos triatomíneos, principalmente em relação aos períodos secos e chuvosos ${ }^{38}$. 


\section{INFECÇÃO POR TRIPANOSOMATÍDEOS}

No presente estudo, a taxa de infecção dos triatomíneos da VIII GERES foi de 2,0\%, menor que a taxa do estado de Pernambuco no biênio 2006-200733 $(9,8 \%)$ e em $2012^{34}$ (21,3\%). P. lutzi apresentou a maior taxa de positividade para flagelados morfologicamente semelhantes a T. cruzi $(38,0 \%)$, que é considerada alta e já foi demonstrada por Silva et al. ${ }^{33,34}$, Ministério da Saúde ${ }^{39}$ e Vinhaes e Dias ${ }^{40}$, com taxas de infecção de $17,8 \%, 35,1 \%, 29,4 \%$ e 2,6\%, respectivamente. Costa et al. ${ }^{35}$ também encontraram $P$. lutzi, porém não calcularam a taxa de infecção. Provavelmente, um dos motivos das altas taxas de infecção apresentadas pelo P. lutzi seja a sua ampla variedade de fontes alimentares: roedores, gambás, tatus, quatis, lagartos, anuros, aves, bovinos, equinos, caprinos, ovinos, suínos, gatos, cães e humanos ${ }^{41,42}$

Neste estudo, T. brasiliensis foi o triatomíneo mais abundante, apresentando taxa de positividade de 1,7\%. Essa espécie foi encontrada positiva para flagelados morfologicamente semelhantes a T. cruzi, com taxas de $6,5 \%^{33}, 19,2 \%^{34}, 2,7 \%{ }^{39}, 1,44 \%^{40}$ e $6,7 \%{ }^{43}$. Todavia, Costa et al. ${ }^{35}$ não encontraram T. brasiliensis infectados nos municípios de Jardim e Caririaçu, no Ceará.

T. sordida apresentou a terceira maior taxa de infecção. Essa espécie já apresentou taxas de infecção relatadas em outros estudos: $0,8 \%{ }^{39}, 0,85 \%^{40}, 2,3 \%^{43}$ e $5,5 \% 44$. Entretanto, nos estudos realizados por Silva et al. ${ }^{33,34}$ e Costa et al. ${ }^{35}$, não foi encontrada infectada.

T. pseudomaculata foi encontrada infectada em 0,9\% dos exemplares coletados, valor abaixo do apresentado por outros estudos: $8,0 \%{ }^{33}, 13,1 \%^{34}, 2,1 \%{ }^{39}, 1,29 \%^{40}$ e $1,7 \%{ }^{43}$. Costa et al. ${ }^{35}$ também registraram espécimes positivos; contudo, a taxa de infecção não foi calculada.

A positividade de $P$. megistus nos estudos de Silva et al. ${ }^{33,34}$, Ministério da Saúde ${ }^{39}$, Vinhaes e $\operatorname{Dias}^{40}$ e Silveira ${ }^{43}$ contrasta com o resultado negativo encontrado no presente estudo, assim como no de Costa et al. ${ }^{35}$. De forma semelhante, espécimes de $R$. neglectus foram encontrados infectados nos estudos conduzidos por Silva et al. ${ }^{33}$, Ministério da Saúde ${ }^{39}$, Vinhaes e Dias ${ }^{40}$ e Silveira ${ }^{43}$, mas não foi o caso deste estudo nem no de Camargo et al. ${ }^{44}$.

Tendo em vista que foram encontrados triatomíneos positivos no presente estudo, é aconselhável que a população seja informada e sensibilizada para a manutenção das medidas de vigilância. Ressalta-se que os casos da doença de Chagas aguda no Brasil ocorrem, na sua maioria, em populações com um a nove anos de escolaridade ${ }^{7}$. Como condição crônica, a morbimortalidade sobressai nas populações mais vulneráveis do ponto de vista econômico e social ${ }^{45}$.

É importante ressaltar a necessidade da manutenção da vigilância entomológica nos municípios por meio das visitas domiciliares realizadas pelos $A C E$, visando à redução da infestação. Realizar melhorias nas habitações, como substituir folhas de palmeira dos telhados e coberturas, tapar frestas e rachaduras nas paredes, são medidas que visam a impossibilitar a formação de colônias de triatomíneos nos domicílios. $\bigcirc$ controle químico e a realização de ações de educação em saúde nas comunidades contribuem para a melhoria da condição de vida da população nos municípios com a presença do vetor transmissor da doença.

Como tratou-se de um estudo com dados secundários, não foi possível fazer um acompanhamento de pacientes diagnosticados com doença de Chagas nos municípios da VIII GERES, no período da presente pesquisa, bem como a realização de uma entrevista com os moradores que relataram a presença de triatomíneos em seus respectivos domicílios.

\section{CONCLUSÃO}

A maioria das espécies coletadas na VIII GERES de Pernambuco são comuns no bioma Caatinga. $\bigcirc$ presente estudo contribui com a vigilância em saúde, pelo fato de ter encontrado espécies positivas em todos os municípios do Sertão de São Francisco, sinalizando - risco de transmissão vetorial, com destaque para P. lutzi que apresentou a maior taxa de infecção para T. cruzi e os municípios de Dormentes e Petrolina que apresentaram as maiores taxas de positividade da VIII GERES.

\section{CONFLITOS DE INTERESSE}

Os autores declaram que não houve conflitos de interesse.

\section{CONTRIBUIÇÃO DOS AUTORES}

Todos os autores cooperaram com a realização da pesquisa, análise e interpretação dos dados e com a redação do manuscrito. Declaram-se responsáveis pelo conteúdo total do artigo, garantindo sua precisão e integridade.

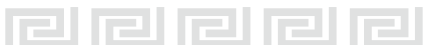

\section{REFERÊNCIAS}

1 Meza Acosta G, Cerecetto Meyer $H$. Seroprevalencia de la enfermedad de Chagas en embarazadas del departamento de Cordillera en el período 2010-2016 y el comportamiento de la seroprevalencia después de 21 años de la implementación del Programa de Control Prenatal de Chagas. Mem Inst Investig Cienc Salud. 2019 dic; $17(3): 10-9$.
2 Argolo AM, Felix M, Pacheco R, Costa J. Doença de Chagas e seus principais vetores no Brasil. Rio de Janeiro: Imperial Novo Milênio; 2008.

3 Galvão C, Jurberg J. Introdução. In: Galvão C, organizador. Vetores da doença de Chagas no Brasil. Curitiba: Sociedade Brasileira de Zoologia; 2014. p. 5-9. (Série Zoologia: guias e manuais de identificação). 
4 Gullan PJ, Cranston PS. Os insetos - um resumo de entomologia. 4. ed. São Paulo: Roca; 2012.

5 World Health Organization. Chagas disease (also known as American trypanosomiasis) [Internet]. Genebra: World Health Organization; 2018 [cited 2018 May 8]. Available from: http://www.who.int/ en/news-room/fact-sheets/detail/chagas-disease-(a merican-trypanosomiasis).

6 Ministério da Saúde (BR). Sistema de Informação de Mortalidade. Mortalidade - Brasil [Internet]. Brasília: SIM; 2019. [citado 2021 jun 21]. Disponível em: http://tabnet.datasus.gov.br/cgi/ tabcgi.exe? sim/cnv/obt10uf.def.

7 Ministério da Saúde (BR). Secretaria de Vigilância em Saúde. Doença de Chagas aguda e distribuição espacial dos triatomíneos de importância epidemiológica, Brasil 2012 a 2016. Bol Epidemiol. 2019 jan;50(2):1-10.

8 Silva MBA, Menezes KR, Farias MCG, Souza Filho IB, Jurberg J. Vigilância entomológica dos vetores da doença de Chagas no agreste pernambucano. Rev Saude Desenvolv. 2017 abr-jun; 11 (7):229-44.

9 Galvão C. Taxonomia dos vetores da doença de Chagas: da forma à molécula, quase três séculos de história. In: Oliveira J, Alevi KCC, Camargo LMA, Meneguetti DUO, organizadores. Atualidades em medicina tropical no Brasil: vetores. Rio Branco: Stricto Sensu; 2020. Cap. 1; p. 9-37.

10 Farias ADSC, Alves ZF, Silva MBA, Coutinho CBD, Rocha DS. Pesquisa entomológica das espécies de triatomíneos encontradas na zona rural do município de Caruaru, estado de Pernambuco, Brasil, de 2011 a 2012. Rev Pan-Amaz Saude. 2019 mar; 10:e201901593.

11 Sousa DM, Ricardo-Silva AH, Freitas SPC, Costa FAC, Toma HK, Carvalho AA, et al. Chagas disease in Northeast of Brazil: findings from a systematic review of literature. ReonFacema. 2018;4(n. esp):952-64.

12 Silva MBA, Rocha DS, Borba RFB. Triatomíneos sinantrópicos de Pernambuco: biogeografia, técnicas laboratoriais e controle de qualidade. Recife: EDUPE; 2019.

13 Galvão C, Gurgel-Gonçalves RG. Vetores conhecidos no Brasil. In: Galvão C, organizador. Vetores da doença de Chagas no Brasil. Curitiba: Sociedade Brasileira de Zoologia; 2014. p. 88-170. (Série Zoologia: guias e manuais de identificação).

14 Jurberg J, Rodrigues JMS, Moreira FFF, Dale C, Cordeiro IRS, Lamas Jr VD, et al. Atlas iconográfico dos triatomíneos do Brasil (vetores da doença de Chagas). Rio de Janeiro: Instituto Oswaldo Cruz; 2014.
15 Silva MBA, Rocha DS, Jurberg J, Silva A, Farias MCG, Galvão C. Registration of new geographical distribution of Panstrongylus geniculatus (Latreille) 1811 (Hemiptera, Reduviidae, Triatominae) in Brazil. Rev Patol Trop. 2016 Jul-Sep;45(3):323-6.

16 Jansen AM, Xavier SCC, Roque ALR. Landmarks of the knowledge and Trypanosoma cruzi biology in the wild environment. Front Cell Infect Microbiol. 2020 Feb; 10:10.

17 Instituto Brasileiro de Geografia e Estatística. Panorama: Pernambuco [Internet]. Rio de Janeiro: IBGE; 2010 [citado 2018 mai 16]. Disponível em: https://cidades.ibge.gov.br/brasil/pe/panorama.

18 Instituto Brasileiro de Geografia e Estatística. Panorama: Afrânio [Internet]. Rio de Janeiro: IBGE; 2010 [citado 2021 mar 6]. Disponível em: https:// cidades.ibge.gov.br/brasil/pe/afranio/panorama.

19 Instituto Brasileiro de Geografia e Estatística. Panorama: Cabrobó [Internet]. Rio de Janeiro: IBGE; 2010 [citado 2021 mar 6]. Disponível em: https://cidades.ibge.gov.br/brasil/pe/cabrobo/ panorama.

20 Instituto Brasileiro de Geografia e Estatística. Panorama: Dormentes [Internet]. Rio de Janeiro: IBGE; 2010 [citado 2021 mar 6]. Disponível em: https://cidades.ibge.gov.br/brasil/pe/dormentes/ panorama.

21 Instituto Brasileiro de Geografia e Estatística. Panorama: Lagoa Grande [Internet]. Rio de Janeiro: IBGE; 2010 [citado 2021 mar 6]. Disponível em: https://cidades.ibge.gov.br/brasil/pe/lagoa-grande/ panorama.

22 Instituto Brasileiro de Geografia e Estatística. Panorama: Orocó [Internet]. Rio de Janeiro: IBGE; 2010 [citado 2021 mar 6]. Disponível em: https:// cidades.ibge.gov.br/brasil/pe/oroco/panorama.

23 Instituto Brasileiro de Geografia e Estatística. Panorama: Santa Maria da Boa Vista [Internet]. Rio de Janeiro: IBGE; 2010 [citado 2021 mar 6]. Disponível em: https://cidades.ibge.gov.br/brasil/ pe/santa-maria-da-boa-vista/panorama.

24 Instituto Brasileiro de Geografia e Estatística. Panorama: Petrolina [Internet]. Rio de Janeiro: IBGE; 2010 [citado 2021 mar 6]. Disponível em: https://cidades.ibge.gov.br/brasil/pe/petrolina/ panorama.

25 Base de Dados do Estado de Pernambuco (BR). Posição geográfica das sedes dos municípios [Internet]. Recife: BDE; 2017 [citado 2021 mar 6]. Disponível em: http://www.bde. pe.gov.br/visualizacao/Visualizacao_formato2. aspx? Codlnformacao $=280 \& \operatorname{Cod}=1$.

26 Base de Dados do Estado de Pernambuco (BR). Relação dos municípios, por região de desenvolvimento [Internet]. Recife: BDE; 2000 [citado 2018 jun 4]. Disponível em: http://www. bde.pe.gov.br/visualizacao/Visualizacao_formato2. aspx? Codlnformacao $=798 \& \operatorname{Cod}=1$. 
27 Base de Dados do Estado de Pernambuco (BR). Cobertura vegetal primitiva [Internet]. Recife: BDE; 2016 [citado 2018 jun 4]. Disponível em: http:// www.bde.pe.gov.br/visualizacao/Visualizacao formato2. aspx? Codlnformacao $=634 \& \operatorname{Cod}=1$.

28 Base de Dados do Estado de Pernambuco (BR). Área dos municípios [Internet]. Recife: BDE; 2016 [citado 2018 jun 4]. Disponível em: http://www. bde.pe.gov.br/visualizacao/Visualizacao formato2. aspx? Codlnformacao $=272 \& \operatorname{Cod}=3$.

29 Base de Dados do Estado de Pernambuco (BR). Tipologia climática [Internet]. Recife: BDE; 2017 [citado 2018 jun 4]. Disponível em: http://www. bde.pe.gov.br/visualizacao/Visualizacao_formato2. aspx? Codlnformacao $=633 \&$ Cod $=1$.

30 Lent $H$, Wygodzinsky P. Revision of triatominae (Hemiptera, Reduviidae), and their significance as vectors of Chagas' disease. Bull Am Mus Nat Hist. $1979 ; 163(3): 123-520$.

31 Galvão C, Dale C. Chaves de identificação para adultos. In: Galvão C, organizador. Vetores da doença de Chagas no Brasil. Curitiba: Sociedade Brasileira de Zoologia; 2014. Cap. 9; p. 171-208. (Série Zoologia: guias e manuais de identificação).

32 Organización Panamericana de la Salud. Guía para muestreo em actividades de vigilancia y control vectorial de la enfermedad de Chagas. Montevidéu (URU): Ed OPS/DPC/CD/276/03; 2003.

33 Silva MBA, Barreto AVMS, Silva HA, Galvão C, Rocha D, Jurberg J, et al. Synanthropic triatomines (Hemiptera, Reduviidae) in the state of Pernambuco, Brazil: geographical distribution and natural Trypanosoma infection rates between 2006 and 2007. Rev Soc Bras Med Trop. 2012 Feb;45(1):60-5.

34 Silva MBA, Menezes KR, Siqueira AM, Balbino VQ, Lorosa ES, Farias MCG, et al. Importância da distribuição geográfica dos vetores da doença de Chagas em Pernambuco, Brasil, em 2012. Rev Patol Trop. 2015 abr-jun;44(2):195-206.

35 Costa ARS, Vieira MS, Pinto LC, Candido AS, Ferreira RJ. Infecção por Trypanosoma cruzi em triatomíneos capturados em dois municípios sul-cearenses. Rev Saude. 2017; 11 (1-2):19-32.
36 Coura JR, Dias JCP. Epidemiology control and surveillance of Chagas disease: 100 years after its discovery. Mem Inst Oswaldo Cruz. 2009 Jul;104 Suppl 1:31-40.

37 Ferro e Silva AM, Sobral-Souza T, Vancine $M H$, Muylaert RL, Abreu AP, Pelloso SM, et al. Spatial prediction of risk areas for vector transmission of Trypanosoma cruzi in the State of Paraná, southern Brazil. PloS Negl Trop Dis. 2018 Oct;12(10): e0006907.

38 Vasconcelos ASOB. Índice de infestação e infecção de triatomíneos por Trypanosoma cruzi na região sudeste do estado do Ceará [dissertação]. Fortaleza (CE): Universidade Federal do Ceará, Faculdade de Farmácia, Odontologia e Enfermagem; 2013. 75 p.

39 Ministério da Saúde (BR) Secretaria de Vigilância em Saúde. Doença de Chagas aguda no Brasil: série histórica de 2000 a 2013. Bol Epidemiol. 2015;46(21):1-9.

40 Vinhaes MC, Dias JCP. Doença de Chagas no Brasil. Cad Saude Publica. 2000;16 supl 2:7-12.

41 Caranha L, Lorosa ES, Rocha DS, Jurberg J, Galvão C. Estudo das fontes alimentares de Panstrongylus lutzi (Neiva \& Pinto, 1923) (Hemiptera: Reduviidae: Triatominae) no Estado do Ceará. Rev Soc Bras Med Trop. 2006 ago;39(4):347-51.

42 Silva MBA, Menezes KR, Farias MCG, Andrade MS, Victor CCA, Lorosa ES, et al. Description of the feeding preferences of triatominae in the Chagas disease surveillance study for the State of Pernambuco, Brazil (Hemiptera: Reduviidae). Rev Soc Bras Med Trop. 2017 Jul-Aug;50(4):543-6.

43 Silveira AC. O inquérito triatomínico (1975-1983). Rev Soc Bra Med Trop. 2011 ;44 supl 2:26-32.

44 Camargo FA, Romano CA, Elias CN, Silva HHG, Silva, IG. Sinanthropization of triatomines (Hemiptera: Reduviidae) in the Itumbiara municipality, Goiás, Brazil. Rev Patol Trop. 2017 Oct-Dec;46(4):339-42.

45 Souza RCM, Diotaiuti L. Desafios para o controle de triatomíneos no Brasil. In: Oliveira J, Alevi KCC, Camargo LMA, Meneguetti DUO, organizadores. Atualidades em medicina tropical no Brasil: vetores. Rio Branco: Stricto Sensu; 2020. Cap. 5; p. 85-98. 